TITLE:

\title{
Parametric dependence of the Pollicott-Ruelle resonances for sawtooth maps
}

$\operatorname{AUTHOR}(S)$ :

Sano, Mitsusada M.

CITATION:

Sano, Mitsusada M.. Parametric dependence of the Pollicott-Ruelle resonances for sawtooth maps. Physical Review E 2002, 66 (4(Part 2)): 046211.

ISSUE DATE:

2002-10

URL:

http://hdl.handle.net/2433/59078

RIGHT:

Copyright 2002 American Physical Society 
PHYSICAL REVIEW E 66, 046211 (2002)

\title{
Parametric dependence of the Pollicott-Ruelle resonances for sawtooth maps
}

\author{
Mitsusada M. Sano \\ Department of Fundamental Sciences, FIHS, Kyoto University, Sakyo, Kyoto, 606-8501, Japan
}

(Received 3 June 2002; published 21 October 2002)

\begin{abstract}
The Pollicott-Ruelle resonances for the sawtooth map are investigated. We turn our attention to the parametric dependence of them with respect to the bifurcation parameter $K$. It is numerically shown that the resonances move in an erratic way if the bifurcation parameter $K$ is supposed to be time. At certain rational values of $K$, it is observed that some resonances shrink to $z=0$. In particular, at positive integer values of $K$ which correspond to the Arnold cat map, all resonances except $z=1$ (i.e., the equilibrium state) shrink to $z$ $=0$. This peculiar behavior is rigorously proved in the Appendix. In addition, the diffusion coefficient of this map is numerically calculated in a very accurate way by evaluating the leading resonance.
\end{abstract}

DOI: 10.1103/PhysRevE.66.046211

PACS number(s): 05.45.Ac, 05.20.-y, 05.45.Mt

\section{INTRODUCTION}

Recent investigation in nonlinear science has revealed an essential role of chaotic motion in nonequilibrium statistical mechanics $[1,2]$. A major development is the discovery of a link between dynamical quantities (Lyapunov exponents, Kolmogorov-Sinai entropy, etc.) and transport coefficients (diffusion coefficient, thermal conductivity, etc.), and the characterization of the steady state (fluctuation theorem). The development has been made for both Hamiltonian dynamical systems and non-Hamiltonian dynamical systems (i.e., NoséHoover thermostat and Gaussian thermostat systems). Active research is still going on for both types of systems.

For Hamiltonian dynamical systems, the time evolution of the probability distribution in the phase space is governed by the Liouville equation. However, in order to investigate the statistical behavior of a given Hamiltonian dynamical system, the Frobenius-Perron equation or the Frobenius-Perron operator is much more suitable than the Liouville equation. The statistical nature of a given system is determined by the spectral property of the Frobenius-Perron operator. The main development has been given by mathematical results in the 1980 's. It has been shown that for hyperbolic dynamical systems, the decay rate to the equilibrium state is well defined and exists $[3,4]$. Now the decay rates $\left\{s_{n}\right\}$ to the equilibrium state are called the Pollicott-Ruelle resonances, which correspond to the eigenvalues $\left\{z_{n}=e^{s_{n}}\right\}$ of the Frobenius-Perron operator. Furthermore, for the systems having the extended space, the leading Pollicott-Ruelle resonances is related to the hydrodynamic mode. Its wave number dependence determines the transport coefficient for a given system, i.e., the diffusion coefficient [5]. However, although theoretical development has been made, an exact calculation of the Pollicott-Ruelle resonances even for area-preserving maps is unfortunately very limited, for instance, the baker map [6].

Recently, this mathematical well-established result has been numerically tested for various systems including a more general situation, i.e., nonhyperbolic systems such as the kicked rotor [7-9], the kicked top [10], and the perturbed cat map [11]. It has been numerically shown that even in the nonhyperbolic regime, if the perturbation parameter is large enough, the concept of the Pollicott-Ruelle resonances persists in nonhyperbolic systems and even the leading reso- nance gives a nice approximation of the diffusion coefficient for the kicked rotor.

The Pollicott-Ruelle resonances are essential not only in classical dynamics but also in quantum dynamics. In semiclassical study of quantum chaotic systems, the PollicottRuelle resonances play an important role for the semiclassical derivation of a two-point energy level correlator $[12,13]$. Furthermore, it has been numerically shown that the eigenvalues of the quantum von Neumann-Liouville operator for the quantum baker map approach to the Pollicott-Ruelle resonances for the classical baker map in the semiclassical limit $\hbar \rightarrow 0$ [14]. For this observation, general consideration is given in [15].

In this paper we give another example whose PollicottRuelle resonances are relatively easily calculated in a numerical sense. We treat the sawtooth map which is hyperbolic in certain parameter regime and which includes some family of the Arnold cat maps [16]. The semiclassical behavior and the property of periodic orbits for the sawtooth map are investigated by the author $[17,18]$. In Sec. II we shall focus our attention on the parametric dependence of the Pollicott-Ruelle resonances for the sawtooth map with respect to the bifurcation parameter $K$ which will be defined below. We shall see the erratic behavior of the PollicottRuelle resonances with respect to $K$. When $K$ is a positive integer, we shall numerically observe that the PollicottRuelle resonances infinitely degenerate except the leading one. This observation is rigorously proved by using the periodic orbit theory in the Appendix. In Sec. III we shall numerically calculate the diffusion coefficient for the sawtooth map. In Sec. IV, we summarize the results.

\section{SAWTOOTH MAP}

The sawtooth map is a generalization of the Arnold cat map, which is defined by

$$
\begin{aligned}
& x_{n+1}=x_{n}+y_{n+1} \quad \bmod 1, \\
& y_{n+1}=y_{n}+K x_{n} \quad \bmod 1,
\end{aligned}
$$

where $x_{n}, y_{n} \in \mathcal{D}, \mathcal{D}=\left[-\frac{1}{2}, \frac{1}{2}\right][16] . K$ is the bifurcation parameter which is real valued. If we introduce winding numbers $w_{y}^{(n)}, w_{x}^{(n)} \in \mathbb{Z}$, the map of Eq. (1) is rewritten as 


$$
\begin{gathered}
x_{n+1}=(1+K) x_{n}+y_{n}-w_{x}^{(n)}-w_{y}^{(n)} \equiv u\left(x_{n}, y_{n}\right), \\
y_{n+1}=y_{n}+K x_{n}-w_{y}^{(n)} \equiv v\left(x_{n}, y_{n}\right) .
\end{gathered}
$$

This representation is useful in the later use.

First, we briefly summarize the behavior of the sawtooth map with respect to the bifurcation parameter $K$. When $K$ $<-4$ and $K>0$, the map is hyperbolic. In this parameter regime, it is mathematically proved that the decay of correlation is exponential [19]. Other mathematical results are found in [20]. If $K$ is a positive integer, the map becomes the Arnold cat map. Many properties of the Arnold cat map are shared when $K$ is positive integer. The most attractive property of the sawtooth map is that if the map is defined in a cylinder or an extended phase space, chaotic diffusion is observed $[16,21,22]$. We shall investigate it in Sec. III. When $-4 \leqslant K \leqslant 0$, the map is stable. The phase space is filled by many elliptic islands.

The time evolution of the probability distribution in the phase space is governed by the Frobenius-Perron equation. If we denote the probability distribution in the phase space at the $n$th time step by $\rho(x, y ; n)$, then it defined by

$$
\rho(X, Y ; n+1)=\int_{-1 / 2}^{1 / 2} d x \int_{-1 / 2}^{1 / 2} d y \mathcal{L}(X, Y ; x, y) \rho(x, y ; n),
$$

where the integral kernel $\mathcal{L}$ is called the Frobenius-Perron operator

$$
\mathcal{L}(X, Y ; x, y)=\delta(X-u(x, y)) \delta(Y-v(x, y)),
$$

where $u(x, y)$ and $v(x, y)$ are the right-hand side (rhs) of Eq. (2).

Our aim is to solve an eigenvalue problem:

$$
\int_{-1 / 2}^{1 / 2} d x \int_{-1 / 2}^{1 / 2} d y \mathcal{L}(X, Y ; x, y) \rho(x, y)=e^{s} \rho(X, Y)
$$

or symbolically

$$
\mathcal{L} \rho=e^{s} \rho .
$$

We sometimes set $z=e^{s}$. The quantities $\left\{s_{n}\right\}_{n=0}^{\infty}$, which are related to the eigenvalues $\left\{z_{n}=e^{s_{n}}\right\}$, are called the PollicottRuelle resonances. Although in this section we directly diagonalize the operator $\mathcal{L}$, another analysis exists. It uses the following Fredholm determinant:

$$
Z(s)=\operatorname{det}\left(1-e^{-s} \mathcal{L}\right)
$$

By the relation $\operatorname{det}(1-A)=\exp [\operatorname{tr} \ln (1-A)]$, the determinant is rewritten to

$$
\begin{aligned}
Z(s) & =\exp \left[\operatorname{tr} \ln \left(1-z^{-1} \mathcal{L}\right)\right] \\
& =\exp \left[-\sum_{n=1}^{\infty} \frac{z^{-n}}{n} \operatorname{tr}\left(\mathcal{L}^{n}\right)\right]
\end{aligned}
$$

$\operatorname{tr}\left(\mathcal{L}^{n}\right)$ can be evaluated as the sum over the contributions of the periodic points [23]. This method is fully used in the Appendix for the system when $K$ is positive integer.

The matrix representation for the Frobenius-Perron operator $\mathcal{L}$ is achieved by using the Fourier basis set $\left\{e^{2 \pi i n x}\right\}_{n=-\infty}^{\infty}$,

$$
\begin{aligned}
\mathcal{L}_{k l, m n}= & \int_{-1 / 2}^{1 / 2} d X \int_{-1 / 2}^{1 / 2} d Y \int_{-1 / 2}^{1 / 2} d x \int_{-1 / 2}^{1 / 2} d y \mathcal{L}(X, Y ; x, y) \\
& \times e^{2 \pi i(k X+l Y-m x-n y)},
\end{aligned}
$$

where $k, l, m, n \in \mathbb{Z}$. The integral is explicitly performed and yields

$$
\mathcal{L}_{k l, m n}=\alpha(k, l, m, n) \beta(k, l, n),
$$

with

$\alpha(k, l, m, n)=\left\{\begin{array}{l}1 \text { for } K(k+l)+k-m=0, \\ \frac{\sin [\pi\{K(k+l)+k-m\}]}{\pi\{K(k+l)+k-m\}} \text { otherwise }\end{array}\right.$

and

$$
\beta(k, l, n)= \begin{cases}1 & \text { for } k+l-n=0, \\ 0 & \text { otherwise }\end{cases}
$$

In Fig. 1 we depict the numerical result of direct diagonalization of the matrix $\mathcal{L}_{k l, m n}$. The matrix size is 121 $\times 121$. The matrix is banded and very sparse and its elements take zero or finite value. Thus, the convergence of eigenvalues is quite good. Figure 1(a) is the result for $K$ $=1.00$ which corresponds to the Arnold cat map. Except the leading resonance $z=1$, all resonances degenerate at $z=0$. This would be related to the number theoretical anomaly of the Arnold cat map as reported in [24]. This behavior shall be investigated in detail in the Appendix. Figure 1(b) is the result for $K=1.30$. In this case, anomalous degeneracy seems to be released. The Pollicott-Ruelle resonances are distributed in the unit circle and are paired with the complex conjugate one. In order to examine the parametric dependence of the Pollicott-Ruelle resonances, we plot the absolute value of the Pollicott-Ruelle resonance versus $K$ in Fig. 2 . It is clearly noticed that some resonances are rapidly moving if $K$ is supposed to be time. The most drastic case is the case when $K$ is a positive integer (the Arnold cat map). All resonances except $z=1$ shrink to $z=0$. Besides this case, when $K$ is certain rational, it is observed that some resonances are falling to $z=0$. Furthermore, the rapid moving resonances are bounded by the circle with a well-defined radius. Unfortunately, we do not know the reason why some resonances move so rapidly. For the case when $K$ is a positive integer, we can explain the behavior of the resonances. In the Appendix we shall prove that the resonances for the Arnold cat map infinitely degenerate, except the leading one. 

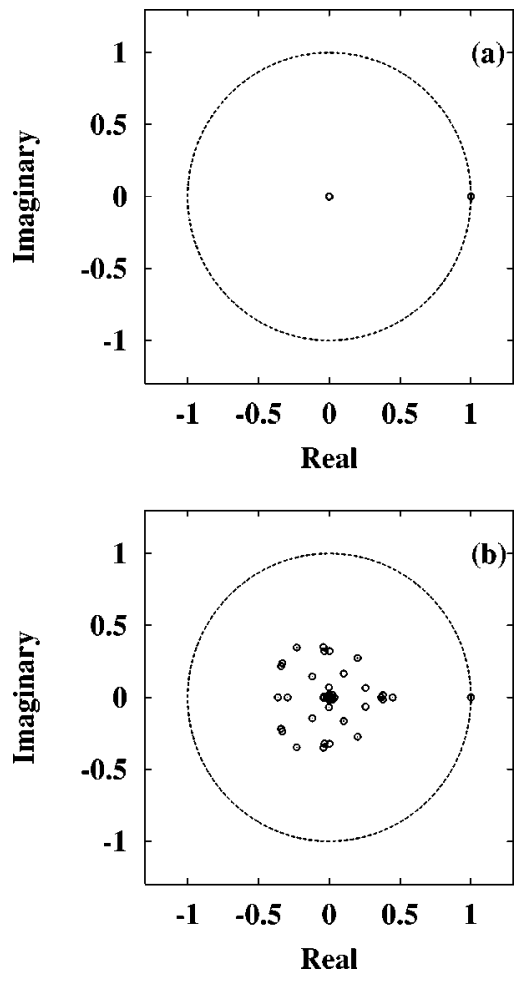

FIG. 1. Pollicott-Ruelle resonances $\left\{z_{n}=e^{s_{n}}\right\}$ : The unit circle is drawn with the dotted line. (a) $K=1.00$. The map is the Arnold cat map. (b) $K=1.30$.

\section{DIFFUSION COEFFICIENT}

We only consider the diffusion along the $y$ axis. The hydrodynamic mode can be examined by the leading PollicottRuelle resonance of the following weighted FrobeniusPerron operator.

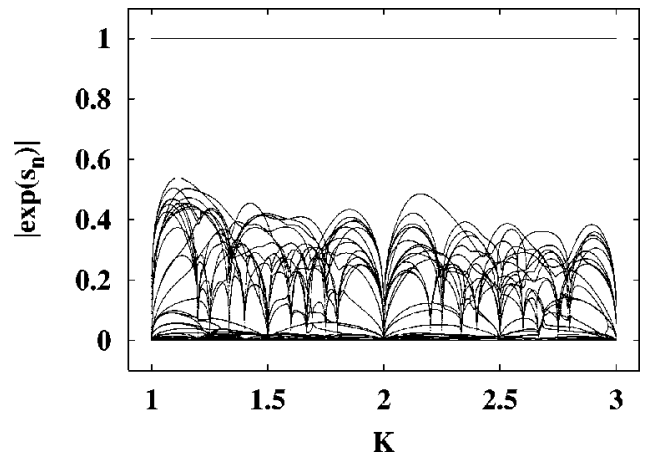

FIG. 2. Parametric dependence of Pollicott-Ruelle resonances: When $K=1,2,3$, all resonances except $z=1$ shrink to $z=0$. For some rational values, some resonances shrink to $z=0$. Rapidly moving resonances seem to be bounded in a circle with a welldefined radius.

$$
\mathcal{L}\left(X, Y ; x, y ; k_{y}\right)=e^{i k_{y} \Delta y} \delta(X-u(x, y)) \delta(Y-v(x, y)) .
$$

$\Delta y$ is the difference between the initial and final positions of the $y$ component in one step iteration for the extended phase space. From Eq. (2) we read

$$
\Delta y=K x .
$$

The matrix elements of the operator Eq. (13) is given by

$$
\mathcal{L}_{k l, m n}\left(k_{y}\right)=\alpha\left(k, l, m, n ; k_{y}\right) \beta(k, l, n),
$$

with

$$
\alpha(k, l, m, n)=\left\{\begin{array}{l}
1 \text { for } K(k+l)+\frac{k_{y} K}{2 \pi}+k-m=0, \\
\frac{\sin \left[\pi\left\{K(k+l)+\frac{k_{y} K}{2 \pi}+k-m\right\}\right]}{\pi\left\{K(k+l)+\frac{k_{y} K}{2 \pi}+k-m\right\}} \text { otherwise. }
\end{array}\right.
$$

The diffusion coefficient for the $y$ component is

$$
D_{y}=\lim _{n \rightarrow \infty} \frac{1}{2 n}\left\langle\left(y_{n}-y_{0}\right)^{2}\right\rangle .
$$

Here the bracket represents the ensemble average of orbits. $D_{y}$ is related to the leading resonance $s_{0}\left(k_{y}\right)[1,5]$,

$$
D_{y}=-\left.\frac{1}{2} \frac{\partial^{2}}{\partial k_{y}^{2}} s_{0}\left(k_{y}\right)\right|_{k_{y}=0} .
$$

In Fig. 3 we depict the $K$-dependence of the diffusion coefficient $D_{y}$. The dotted line represents the curve $D_{y}$ $=K^{2} / 24$. Using the periodic orbit theory, it is shown that when $K$ is positive integer, the diffusion coefficient $D_{y}$ takes this value $K^{2} / 24$ [21,22]. In [21], the diffusion coefficient for other value of $K$ has been numerically evaluated by taking 


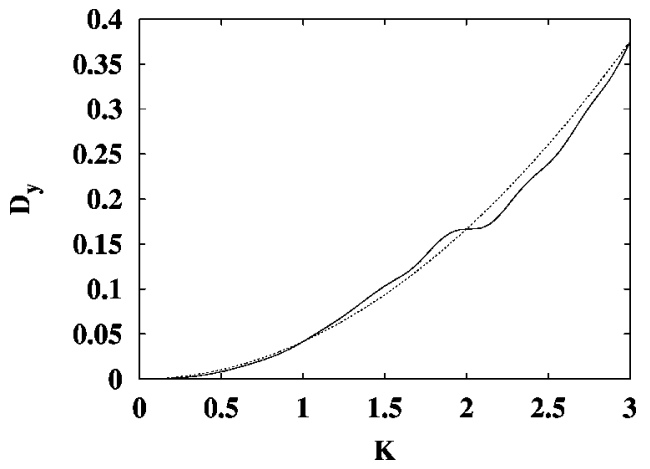

FIG. 3. Parametric dependence of the diffusion coefficient $D_{y}$ : The parabolic curve $\left(K^{2} / 24\right)$ (the dotted line) which is obtained from the periodic orbit theory (exact for the case when $K$ is a positive integer and the approximation for the case when $K$ is a noninteger) is also shown. When $K=1,2,3$ (the Arnold cat map), the value of $D_{y}$ is on the dotted line.

ensemble average of orbits. Then a similar curve has been obtained, but it had large statistical error. Here we have used the explicit evaluation of the diffusions coefficient. We expect that the reliable digits are, at least, five. It is clearly seen that the dotted curve obtained from the periodic orbit theory is a nice approximation for the case when $K$ is a small nonpositive integer. However, when $K$ becomes large away from the positive integer, the deviation from this theoretical curve (the dotted line) seems to become large. In addition, note that the calculated curve seems to be a smooth, not fractal, object.

\section{SUMMARY}

We have calculated the Pollicott-Ruelle resonances for the sawtooth map. It is shown that the off-leading resonances are rapidly moving in an erratic way if the bifurcation parameter $K$ is supposed to be time. In particular, when $K$ is a positive integer, the sudden shrink of resonances (except $z=1$ ) to $z$ $=0$ occurs. This phenomena is rigorously proved in the Appendix. Even when $K$ is certain positive rational, some resonances also shrink to $z=0$. The reason for this behavior is unknown.

Note added in proof. Recently Dr. A. Tanaka followed my numerical calculation and found that the convergence of the second leading resonance with increasing the matrix size is rather bad when $K$ is a noninteger. This problem probably is due to the choice of the basis set. In addition, Professor S. Tasaki showed, in his unpublished work, that the choice of the basis set for the Arnold cat map has a delicate problem.

\section{APPENDIX}

In this appendix we show that the Pollicott-Ruelle resonances for the Arnold cat map have the leading one $z_{0}=e^{s_{0}}$ $=1$, and others $z_{n}=e^{s_{n}}=0$ for $n \geqslant 1$. For simplicity, we consider the following Arnold cat map with $K$ as a positive integer:

$$
\left(\begin{array}{l}
x^{\prime} \\
y^{\prime}
\end{array}\right)=A\left(\begin{array}{l}
x \\
y
\end{array}\right) \bmod 1,
$$

where

$$
A=\left(\begin{array}{cc}
K+1 & 1 \\
K & 1
\end{array}\right) .
$$

For the Arnold cat map, the number of fixed points of order $m$ is precisely given by $\operatorname{det}\left(A^{m}-I\right)$ [24]. The Fredholm determinant of the Arnold cat map is

$$
\begin{aligned}
Z_{\text {cat }}(z) & =\operatorname{det}\left(1-z^{-1} \mathcal{L}\right) \\
& =\exp \left[-\sum_{m=1}^{\infty} \frac{z^{-m}}{m} \operatorname{tr}\left(\mathcal{L}^{m}\right)\right] \\
& =\exp \left[-\sum_{m=1}^{\infty} \frac{z^{-m}}{m} \sum_{p \in \operatorname{Fix}^{m}, m=n_{p} r} \frac{1}{\left|\operatorname{det}\left(J_{p}^{r}-I\right)\right|}\right] \\
& =\exp \left[-\sum_{m=1}^{\infty} \frac{z^{-m}}{m}\right] \\
& =\frac{z-1}{z},
\end{aligned}
$$

where $J_{p}$ is the Jacobian of the Arnold cat map for the primitive periodic point $p$. Fix $A^{m}$ is the set of fixed points of order $m$. We note that $J_{p}^{r}=A^{n} p^{r}=A^{m}$, since the map is linear. $n_{p}$ is the period for the primitive periodic point $p$. From the second line to the third line, we used the fact that $\sum_{p \in \mathrm{Fix} A^{m}, m=n_{p} r} 1$ $=\operatorname{det}\left(A^{m}-I\right)$. Therefore, the Pollicott-Ruelle resonances for the Arnold cat map are $z=1$, corresponding to the invariant density, and the rest is infinitely degenerated at $z=0$. This is what we have observed in Fig. 1(a). The proof is completed.
[1] P. Gaspard, Chaos, Scattering and Statistical Mechanics (Cambridge University Press, Cambridge, 1998).

[2] J.R. Dorfman, An Introduction to Chaos in Nonequilibrium Statistical Mechanics (Cambridge University Press, Cambridge, 1999).

[3] M. Pollicott, Invent. Math. 81, 415 (1985).

[4] D. Ruelle, Phys. Rev. Lett. 56, 405 (1986); J. Stat. Phys. 44, 281 (1986).
[5] P. Cvitanović, J.-P. Eckmann, and P. Gaspard, Chaos, Solitons Fractals 6, 113 (1995).

[6] H.H. Hasegawa and W.C. Saphir, Phys. Rev. A 46, 7401 (1992).

[7] R. Balescu, Statistical Dynamics: Matter out of Equilibrium (Imperial College Press, London, 1997).

[8] R. Balescu, J. Stat. Phys. 98, 1169 (2000).

[9] M. Khodas and S. Fishman, Phys. Rev. Lett. 84, 2837 (2000). 
[10] J. Weber, F. Haake, and P. Šeba, Phys. Rev. Lett. 85, 3620 (2000).

[11] G. Blum and O. Agam, Phys. Rev. E 62, 1977 (2000).

[12] O. Agam, B. Altshuler, and A.V. Andreev, Phys. Rev. Lett. 75, 4389 (1995).

[13] E. Bogomolny and J.P. Keating, Phys. Rev. Lett. 77, 1472 (1996).

[14] S. Fishman, Supersymmetry and Trace Formulae, edited by I. V. Lerner, J.P. Keating, and D.E. Khmelnitskii (Kluwer Academic/Plenum Publishers, New York, 1999), p. 193.

[15] M.M. Sano, Phys. Rev. E 59, R3795 (1999).

[16] I. Percival and F. Vivaldi, Physica D 27, 373 (1987); N. Bird and F. Vivaldi, ibid. 30, 164 (1988).

[17] M.M. Sano, J. Phys. A 29, 6087 (1996).

[18] M.M. Sano, Chaos 10, 195 (2000).

[19] N.I. Chernov, J. Stat. Phys. 69, 111 (1992).

[20] S. Vaienti, J. Stat. Phys. 67, 251 (1992).

[21] I. Dana, Physica D 39, 205 (1989).

[22] R. Artuso and R. Strepparava, Phys. Lett. A 236, 469 (1997).

[23] R. Artuso, E. Aurell, and P. Cvitanović, Nonlinearity 3, 325 (1990); 3, 361 (1990); P. Cvitanoviác, R. Artuso, R. Mainieri, G. Tanner, and G. Vattay, Classical and Quantum Chaos (Niels Bohr Institute, Copenhagen, 2001), www.nbi.dk/ChaosBook/. [24] J.P. Keating, Nonlinearity 4, 277 (1991); 4, 309 (1991). 